\title{
Improvements in discrimination of bulk and trace elements in long- wavelength double pulse LIBS
}

\author{
J. R. Freeman ${ }^{1 *}$, P. K. Diwakar ${ }^{1}$, S. S. Harilal ${ }^{1 * *}$, and A. Hassanein ${ }^{1}$ \\ ${ }^{1}$ Center for Materials Under eXtreme Environment, School of Nuclear Engineering, Purdue \\ University, 500 Central Drive, West Lafayette, Indiana 47907 USA \\ "Present Address: The Pennsylvania State University, University Park, Pennsylvania 16802 USA \\ ** Present Address: Pacific Northwest National Laboratory, Richland, Washington 99352 USA
}

Corresponding author: S. S. Harilal

J. R. Freeman

freeman.justinr@gmail.com

P. K. Diwakar

pdiwakar@purdue.edu

S. S. Harilal

hari@pnnl.gov

A. Hassanein

hassanein@purdue.edu

(C) 2014. This manuscript version is made available under the Elsevier user license 


\begin{abstract}
In this work we study the effectiveness of long-wavelength heating in double pulse (DP) LIBS, quantitatively comparing figures of merit with those from traditional single pulse (SP) LIBS. The first laser pulse serves as the source of sample ablation, creating an aerosol-like plume that is subsequently reheated by the second laser pulse. At power densities used, the long-wavelength $\mathrm{CO}_{2}$ laser pulse does not ablate any of the solid sample in the atmospheric conditions investigated, meaning plasma emission and enhanced signal can be entirely attributed to the reheated plume rather than increased sample ablation. The signal discrimination was improved significantly using long-wavelength DP-LIBS. For bulk elemental analysis, DP-LIBS provided maximum enhancements of about 14 and 15 times for $\mathrm{S} / \mathrm{N}$ and $\mathrm{S} / \mathrm{B}$, respectively, compared to SP-LIBS using the same quantity of ablated sample. For trace elemental analysis, maximum enhancements of about 7 and 4 times for $\mathrm{S} / \mathrm{N}$ and $\mathrm{S} / \mathrm{B}$, respectively, were observed. These improvements are attributed to effective coupling between the second heating pulse and expanding plume and more efficient excitation of plume species than from the single pulse alone. Most significant improvements were observed in the case of low prepulse energy and minimal sample ablation. While bulk elemental analysis observed improvements for all prepulse energies studied, trace element discrimination only significantly improved for the lowest prepulse energy studied.
\end{abstract}

Keywords: Laser-induced breakdown spectroscopy; Double pulse LIBS; Optical emission spectroscopy; Laser-produced plasma. 


\section{Introduction}

Laser-induced breakdown spectroscopy (LIBS) is a technique used to determine the elemental constituents of unknown samples. After irradiation by an intense laser pulse, the spectrum of light emitted from the resultant plasma can be analyzed to determine the makeup of the sample [1-3]. Due to its relatively simple design requiring only line of sight, availability of compact components, analysis capabilities for solids, liquids, and gases, and in situ capabilities, LIBS has gained popularity in a growing number of fields [4-8]. Compared to other elemental detection techniques, LIBS offers advantages, but also currently suffers from several limitations which include semi-destruction of the sample and lack of sensitivity compared to other laboratory based analytical techniques. To improve the capabilities of LIBS, consideration must be given to the two main components; laser excitation source and light collection system. Laser parameters such as wavelength, pulse duration, and energy can affect the ablated sample size and subsequent light emission from the plasma, while spectral resolution and detector gating parameters of the light collection system have significant influences on the resultant analysis. Shorter laser wavelengths are useful for providing higher mass ablation, while longer wavelengths are more effective at heating and exciting the plume through increased inverse bremsstrahlung absorption by electrons [9-11]. Ultrashort fs laser pulses are useful for minimizing the heat affected zone and thermal damage in delicate samples [12]. Improved resolution of the light collection system offers the opportunity to resolve closely spaced emission lines and the possibility to differentiate isotopic shifts for elements [13, 14]. Detector gate delay and gate width prove helpful in minimizing the effects of strong continuum emission at early times in plasma evolution. 
Non-traditional laser-excitation setups have also been explored, including the use of a second laser for reheating of the plasma plume $[15,16]$. This double pulse (DP) LIBS scheme introduces further parameters requiring optimization, but is useful in improving accuracy of measurements by separating the processes of sample ablation and excitation [15]. While DPLIBS studies demonstrated promising results, the cause of increased signal intensity, be it from increased mass ablation or improved laser-plasma coupling, is not always clear [16-21]. Previous studies using a double-pulse technique and long-wavelength reheating to improve extreme ultraviolet emission have proven highly successful [22], so applying a similar technique for LIBS systems may significantly improve the visible emission used for LIBS spectral analysis. Several studies have been done using a long-wavelength $\mathrm{CO}_{2}$ laser in DP-LIBS systems [11,23-26], but there is no one comprehensive study that simultaneously investigates the effects of signal noise and background levels, gating parameter optimization, first-pulse energy and ablated mass variation, analysis of trace elements, and also highlights the benefit of the $\mathrm{CO}_{2}$ laser increasing emission without increasing sample destruction.

In this paper we applied the DP-LIBS technique using a long-wavelength second pulse to address shortcomings in LIBS studies. A traditional SP-LIBS experiment was performed to provide a benchmark for subsequent DP experiments. For the DP-LIBS analysis, a shorter wavelength prepulse evaluated at several laser energies was used to ablate material from the sample, while the second long-wavelength pulse was used to excite the plume without further increasing sample ablation, which is often a major contributing factor of improved signal in collinear Nd:YAG-based DP-LIBS studies. Absolute signal intensity alone is not an adequate indicator of improved system performance and element discrimination, so signal-to-noise ( $\mathrm{S} / \mathrm{N})$ and signal-to-background (S/B) ratios were calculated and used to compare spectral quality and 
performance metrics in order to provide a quantitative understanding of the improvements offered by long-wavelength heating in DP-LIBS.

\section{Experimental Setup}

A schematic of the experimental setup is provided in Figure 1. The target used in all experiments was a brass sample. LA-ICP-MS analysis of the sample was performed to determine approximate elemental compositions of $73 \% \mathrm{Cu}$ and $26 \% \mathrm{Zn}$, with the remainder consisting of trace elements including $\mathrm{Fe}$ and $\mathrm{Pb}$. A Nd:YAG laser operating at its fundamental wavelength of $1.064 \mu \mathrm{m}$ was used as the first pulse for sample ablation at an incident angle of $5^{\circ}$ off-normal to the target. A small off-normal angle of incidence for the first pulse was required to avoid different laser focusing optics required for the two lasers. Spot size of this first pulse was maintained at $\sim 1 \mathrm{~mm}$ diameter and laser energy was varied from 2-100 mJ with $10 \mathrm{~ns}$ full width half maximum (FWHM) pulse duration. The second laser pulse was incident normal to the target surface and pre-plasma plume expansion. This second heating pulse was from a transversely excited atmospheric (TEA) $\mathrm{CO}_{2}$ laser operating at $10.6 \mu \mathrm{m}$. Spot size of this laser was focused to $\sim 3 \mathrm{~mm}$ diameter to compensate for radial expansion of the ablation plume from the first laser

pulse and $\mathrm{CO}_{2}$ laser pulse energy was maintained at $400 \mathrm{~mJ}$. Programmable timing generators were used to synchronize both laser pulses and adjust interpulse delay times. Light emitted from the plasma was collected and focused onto an optical fiber using an off-axis parabolic mirrorbased collimator located $50^{\circ}$ off-normal to the target. The fiber optic transferred the light to a 0.75 m Czerny-Turner spectrograph, where the dispersed light spectrum was captured on an ICCD. The spectrograph grating used in these experiments has 150 grooves/mm with nominal 
dispersion of approximately $8.77 \mathrm{~nm} / \mathrm{mm}$, allowing for a large spectral region to be captured from a single laser shot while still offering resolution of $0.36 \mathrm{~nm}$. Gate delays and gate widths for spectral collection were controlled using an internal programmable timing generator built into the ICCD. All experiments were conducted in ambient air atmospheric conditions.

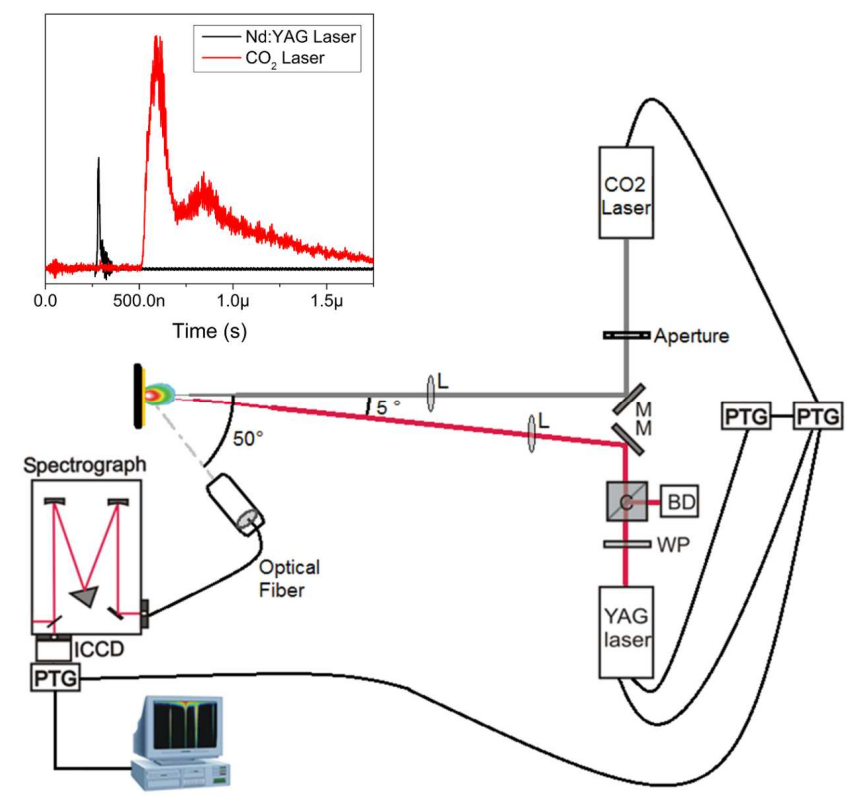

Figure 1 - Schematic of experimental setup (PTG, programmable timing generator; L, lens; ICCD, intensified charge-coupled device; $\mathrm{M}$, mirror; $\mathrm{C}$, cube polarizer; $\mathrm{BD}$, beam dump; $\mathrm{WP}$, waveplate). Temporal profiles of $\mathrm{Nd}: \mathrm{YAG}$ and $\mathrm{CO}_{2}$ laser pulses are provided in the upper-left corner.

\section{Results and Discussion}

To create a benchmark for DP-LIBS experiments, a traditional SP-LIBS analysis was carried out employing a $1.064 \mu \mathrm{m}$ Nd:YAG laser as the excitation source. For DP-LIBS experiments the $1.064 \mu \mathrm{m}$ laser was used for ablation while a $10.6 \mu \mathrm{m} \mathrm{CO} \mathrm{CO}_{2}$ laser was used for reheating. Any mass ablation by the reheating pulse is ruled out due to the low irradiance of the $\mathrm{CO}_{2}$ pulses used in these experiments, coupled with the ambient pressure environment. The 
insignificant ablation by the $\mathrm{CO}_{2}$ laser can also correlate to low plasma critical density which is related to laser wavelength as $n_{e c} \propto \lambda^{-2}$ [27]. Once plasma density exceeds the critical density the plasma can be considered opaque to further laser radiation and subsequent laser energy is deposited in the coronal region or reflected. In an ambient pressure environment this results in shielding of the target and negligible target ablation by the $\mathrm{CO}_{2}$ laser, instead producing excitation almost entirely of the ambient environment. This was confirmed in our experiments through visual inspection of the sample after repeated $\mathrm{CO}_{2}$ laser pulses, indicating negligible target ablation, and analysis of the optical emission, which showed only excited atmospheric gas species and no noticeable excited $\mathrm{Cu}$ or $\mathrm{Zn}$ species from the target in single pulse cases.

\subsection{Performance Metrics}

Increases of absolute line intensity observed in many LIBS studies can be misleading in determining spectral quality and a LIBS system's ability to discriminate an emission signal from noise and background levels. Line intensity can artificially increase with increasing background intensity without necessarily offering improved detection capabilities and the different decay rates for atomic emission and continuum background emission necessitate a more comprehensive means of analysis [28]. In order to address this need and quantitatively compare spectral quality and performance metrics, signal-to-noise ( $\left.\mathrm{S} / \mathrm{N}=\frac{I_{\text {Max }}-I_{\text {Background }}}{\sigma_{\text {Background }}}\right)$ and signal-to-background ( $\mathrm{S} / \mathrm{B}$ $\left.=\frac{I_{\text {Max }}-I_{\text {Background }}}{I_{\text {Background }}}\right)$ ratios have been calculated for certain spectral emission lines to benchmark and compare results. These ratios are calculated from the peak line intensity, $I_{\text {Max }}$, the averaged background intensity near to the line, $I_{\text {Background }}$, and the standard deviation of the background intensities, $\sigma_{\text {Background }} . \mathrm{S} / \mathrm{N}$ and $\mathrm{S} / \mathrm{B}$ trends are often similar, but can occasionally be 
inconsistent due to the transient nature of a signal. The $\mathrm{S} / \mathrm{N}$ ratio is considered the correct ratio on which to base signal optimization [28] because it more effectively quantifies the ability to discriminate a true signal from fluctuations in the data. For this reason it will be used as the primary performance metric throughout this paper.

A representative emission spectrum from the brass sample is provided in Figure 2 and clearly identifiable emission lines have been labeled. It is clear from Figure 2 that there are many relatively intense $\mathrm{Fe}$ lines for a trace component. The $\mathrm{Cu}$ I line at $510.55 \mathrm{~nm}$ was chosen for $\mathrm{S} / \mathrm{N}$ and $\mathrm{S} / \mathrm{B}$ calculations as a representative bulk element and the Fe I line at $423.59 \mathrm{~nm}$ was chosen for calculations as a representative trace element. These lines were chosen because surrounding wavelengths (shorter wavelength regions for the $\mathrm{Cu} \mathrm{I}$ and $\mathrm{Fe} \mathrm{I}$ lines) were absent of emission lines throughout the evolution of the plasma, providing for consistent and reliable background intensities when calculating $\mathrm{S} / \mathrm{N}$ and $\mathrm{S} / \mathrm{B}$ ratios. 


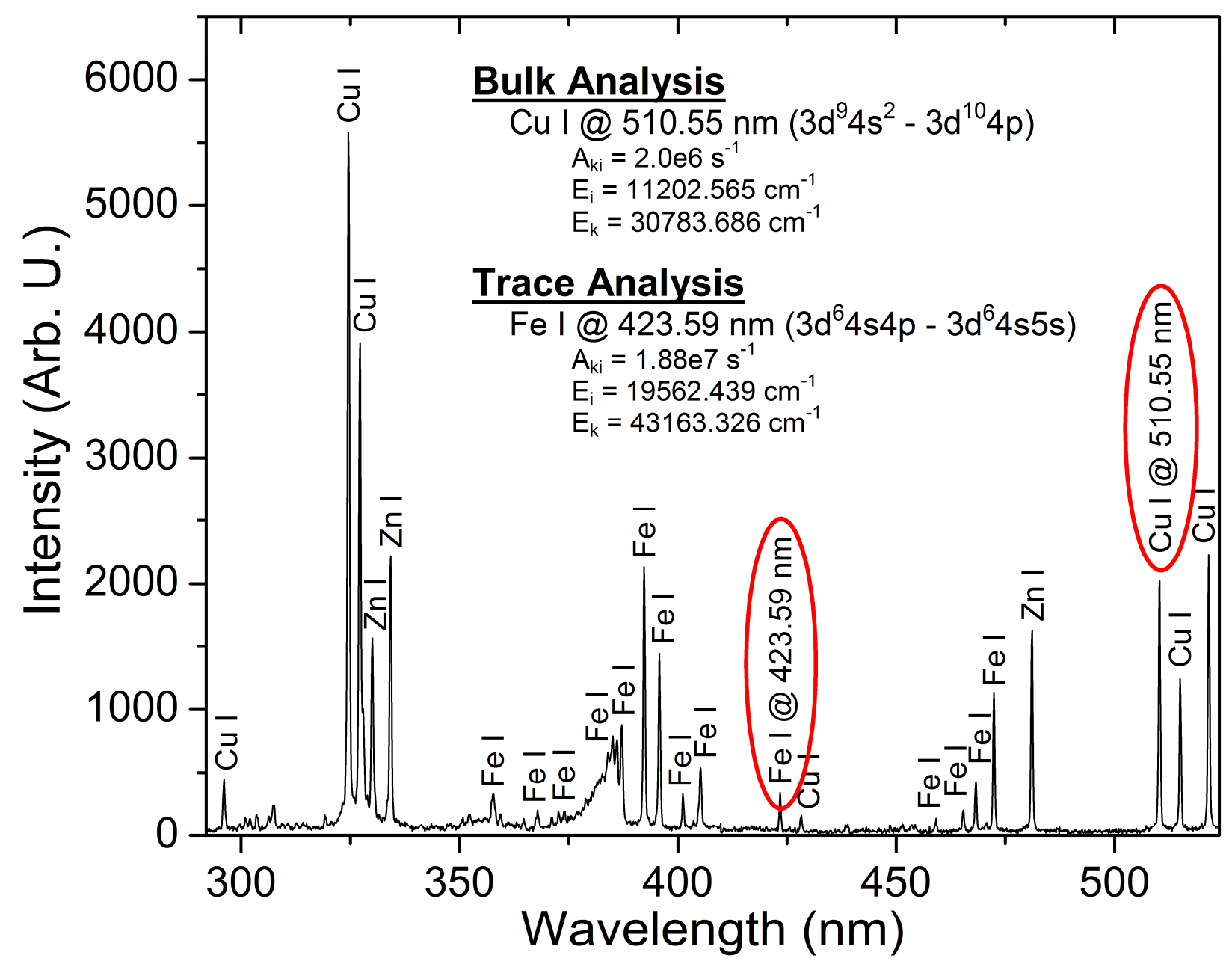

Figure 2 - Representative single-shot double pulse emission spectrum with peaks labeled, lines of interest identified, and transition parameters for lines of interested provided $\left(\mathrm{A}_{\mathrm{ki}}\right.$ : Transition probability, $\mathrm{E}_{\mathrm{i}}$ and $\mathrm{E}_{\mathrm{k}}$ are lower and upper energy levels of selected emission lines).

\subsection{Single pulse LIBS benchmark}

The SP-LIBS benchmarking was done using a $1.064 \mu \mathrm{m} \mathrm{Nd:YAG} \mathrm{laser} \mathrm{in} \mathrm{the} \mathrm{energy}$ range $10-100 \mathrm{~mJ}$ with $1 \mathrm{~mm}$ diameter spot size and gate delay was varied with a fixed $20 \mu$ sate width. By varying the gate delay after the laser pulse, one is able to avoid the high background continuum emission present at early times in plasma evolution. After subtracting the dark current in the detector, light leakage onto the ICCD was found to be negligible, meaning the detector 
could be left open for long gate widths without incurring additional background levels. Plume emission persistence lasted approximately $20 \mu$ s, so the $20 \mu$ s gate width allowed for maximum light collection for all gate delays without accumulating additional background noise. Figure 3 shows the SP-LIBS S/N data at different laser energies as a function of gate delay. Peak gate delay values are similar between bulk and trace elements and in both cases are found to occur at later times with increasing laser energy. This can be explained by the hotter plasma produced by increasing laser energies, requiring a longer time for background emission to decay and excited neutral emission to dominate. The large fluctuations present in the trace element data are due to single pulse emission analysis and attributed to the inherent nature of trace element analysis, where small changes in concentration or plasma properties become magnified. It is worth noting that DP-LIBS results presented in subsequent figures show less significant fluctuations and offer increased consistency. The peak S/N values from Figure 3 are used later for comparison against DP-LIBS results.
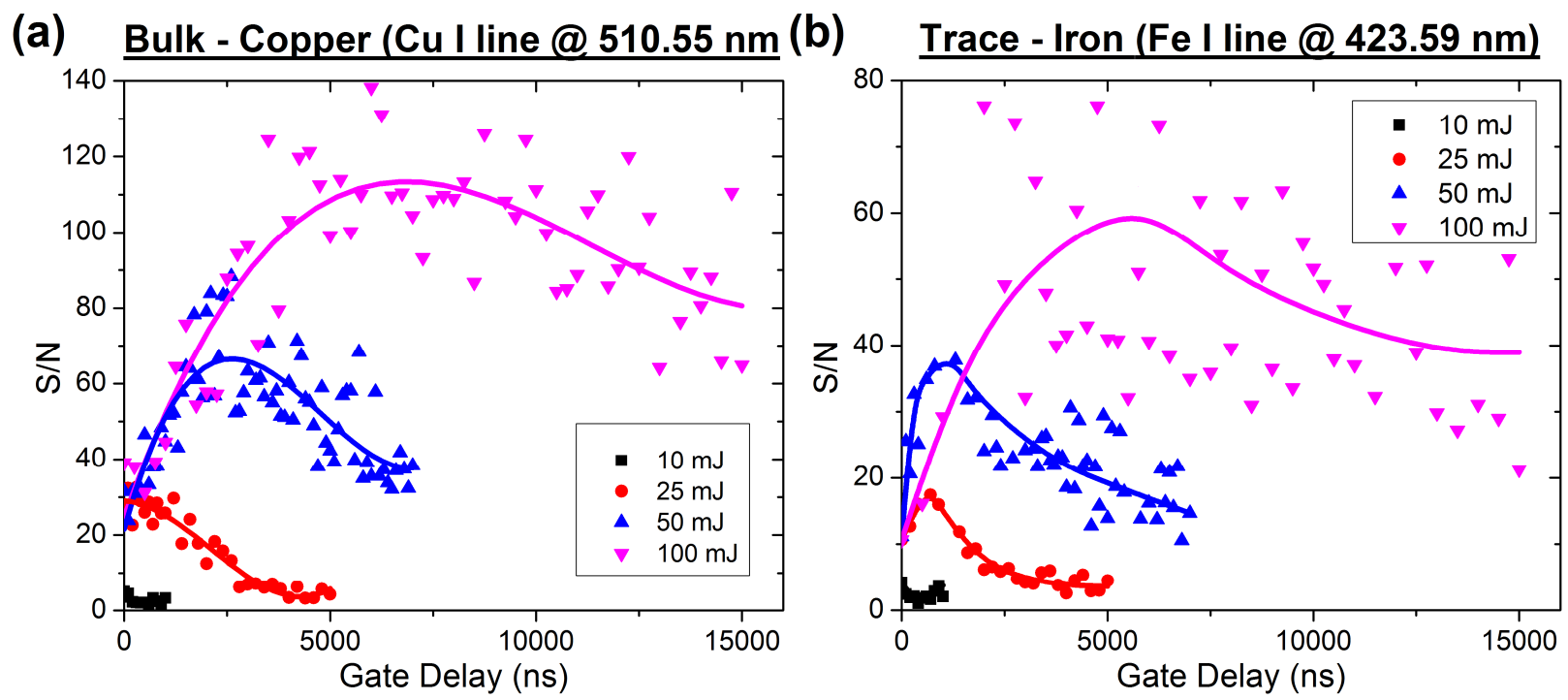

Figure 3 - SP-LIBS: S/N values with respect to gate delay for bulk (a) and trace (b) elements under varying Nd:YAG laser energy. S/B data displayed similar trends and is therefore omitted. 


\subsection{Double pulse LIBS}

Since ablation of the sample from the $\mathrm{CO}_{2}$ laser pulse was found to be negligible, as explained in the previous discussion of plasma critical density, material removal is achieved only through the $1.064 \mu \mathrm{m} \mathrm{Nd:YAG} \mathrm{laser.} \mathrm{A} \mathrm{study} \mathrm{then} \mathrm{presents} \mathrm{itself} \mathrm{to} \mathrm{evaluate} \mathrm{whether} \mathrm{LIBS}$ system performance can be improved by using a second laser pulse which does not ablate additional sample material, but only excites the plume created by the first pulse. Absorption of laser radiation from the second pulse via free electrons in the plume is governed primarily through inverse bremsstrahlung absorption. Inverse bremsstrahlung absorption is related to laser wavelength as [10] $\alpha_{i b} \propto \lambda^{3}$, meaning long-wavelength laser irradiation will be more effective at heating the plume. As the plasma plume created by the Nd:YAG laser pulse expands and cools in the ambient environment, the plume can be thought of as a well-confined bubble consisting of the sample in a vaporized form. The subsequent $\mathrm{CO}_{2}$ laser pulse then heats the newly-created vaporized sample, raising the plume temperature and exciting and ionizing plume species without contributing further material removal from the sample. As Nd:YAG laser energy is increased, more target material is ablated and the electron density in the vaporized bubble also increases, but as time after the first laser pulse increases, the plume expands and density decreases.

Three important parameters in optimizing a DP-LIBS system include prepulse laser energy, the time delay between prepulse and heating pulse, and detector gate timings for light collection. With the current DP-LIBS setup, the persistence of plume emission was observed to last approximately $50 \mu$ s due to plume temperature remaining high for a longer time, so in order to maximize light collection a $50 \mu$ s detector gate width was used throughout DP-LIBS experiments. 
Using a fixed $50 \mu$ s gate width and fixed $2 \mu$ s interpulse delay, Figure 4 shows the effect of varying gate delay on $\mathrm{S} / \mathrm{N}$ values for bulk and trace elements using different prepulse laser energies. SP-LIBS results are also shown in Figure 4 for comparison. For bulk element analysis, a clear trend is evident. With increasing prepulse energy, the peak gate delay occurs at later times and $\mathrm{S} / \mathrm{N}$ values increase, though they appear to plateau beyond $25 \mathrm{~mJ}$ prepulse energy. These observations can be explained by the increased ablation from higher energy prepulses resulting in higher plume density. As plume density increases, the number of excited species increases, but so does the continuum emission, requiring longer gate delays for it to decay. Beyond $25 \mathrm{~mJ}$, the plasma may be approaching the critical density of the $1.064 \mu \mathrm{m} \mathrm{Nd:YAG} \mathrm{laser,} \mathrm{resulting} \mathrm{in} \mathrm{only}$ minor increases in ablated sample mass due to plasma shielding. The plateau evident for prepulse energies above $25 \mathrm{~mJ}$ suggests that coupling efficiency between the second heating pulse and the plume appears to saturate. This saturation effect with increasing pulse energy has been reported before [29].

For trace element analysis, the data indicates a similar trend of increasing peak gate delay with increasing prepulse energy. As prepulse laser energy increases, the improvement offered by DP-LIBS appears to decrease and at $50 \mathrm{~mJ}$ prepulse energy, the use of DP-LIBS shows no clear advantage over SP-LIBS. For low energy SP-LIBS, laser energy is used primarily for sample ablation, and excitation of the plume is inefficient. For higher energy SP-LIBS, along with increased sample ablation, the heating of the plume will be greater. However, because of this limited-mass situation, DP-LIBS will not able to provide any further improvement over the SPLIBS results for trace elements. This indicates that DP-LIBS with long-wavelength heating is most advantageous for trace elemental detection when low prepulse energies and hence, low sample ablation is required, such as the case for delicate samples. 
(a) Bulk - Copper (Cu I line @ 510.55 nm)

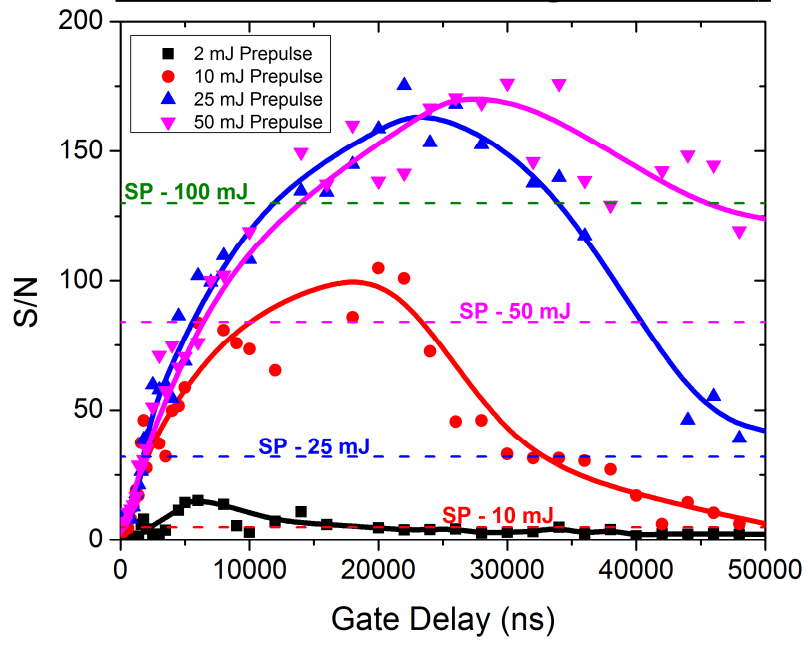

(b) Trace - Iron (Fe I line @ 423.59 nm)

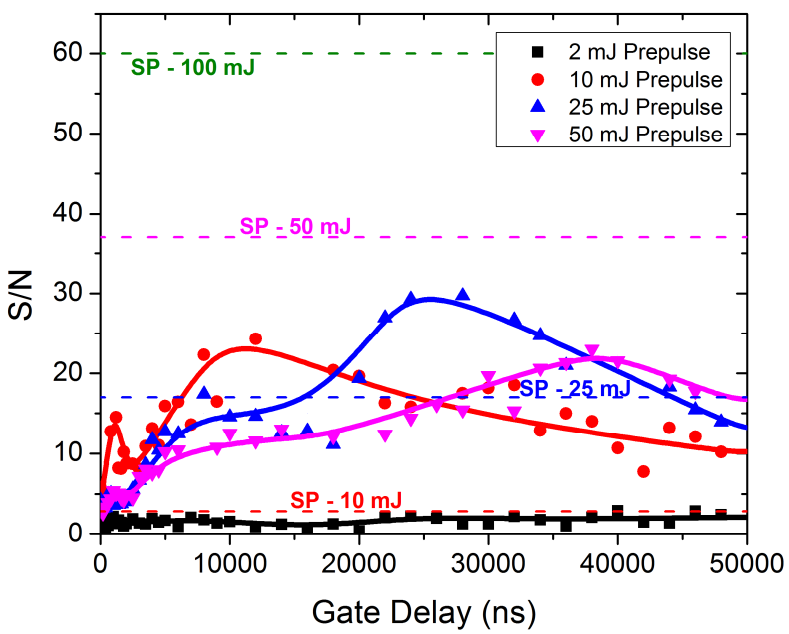

Figure 4 - DP-LIBS: S/N values with respect to gate delay for bulk (a) and trace (b) elements under varying prepulse laser energy. Detector gate width and interpulse delay were fixed for this data. Dashed lines represent optimal SP-LIBS values obtained at indicated laser energies. S/B data displayed similar trends and is therefore omitted.

By varying the timing between the two laser pulses, referred to as the interpulse delay, one is able to control the size and density of the pre-formed plume as it expands before it is heated by the second pulse and experimentally find the plume properties which results in optimal excitation by the second long-wavelength heating pulse. Figure 5 shows the effect of varying the interpulse delay on $\mathrm{S} / \mathrm{N}$ values after the optimal gate delays have already been determined (see Figure 4). SP-LIBS results are also shown in the figures for comparison. We see that after optimizing gate delay, increasing the interpulse delay beyond the initial plasma expansion time produces minor differences for bulk element detection, suggesting that after initial plasma expansion the vaporized plume from the first laser pulse remains relatively stable and does not undergo significant transient behavior over several microseconds. However, the analysis for trace elements shows that even small changes in plume properties during expansion will have a more pronounced effect, as seen by the more complicated trends seen in Figure 5 (b), though 
fluctuations appear less significant than in the SP-LIBS case from Figure 3. From the figures, there does not appear to be a significant difference between peak values from 25 and $50 \mathrm{~mJ}$ prepulse laser energies. Similar to the previous discussion from Figure 4, this may be due to saturation in the coupling efficiency between the second heating pulse and the plume. Again for trace element detection, increasing prepulse energy decreases the advantage offered by DPLIBS.

(a) Bulk - Copper (Cu I line @ 510.55 nm)

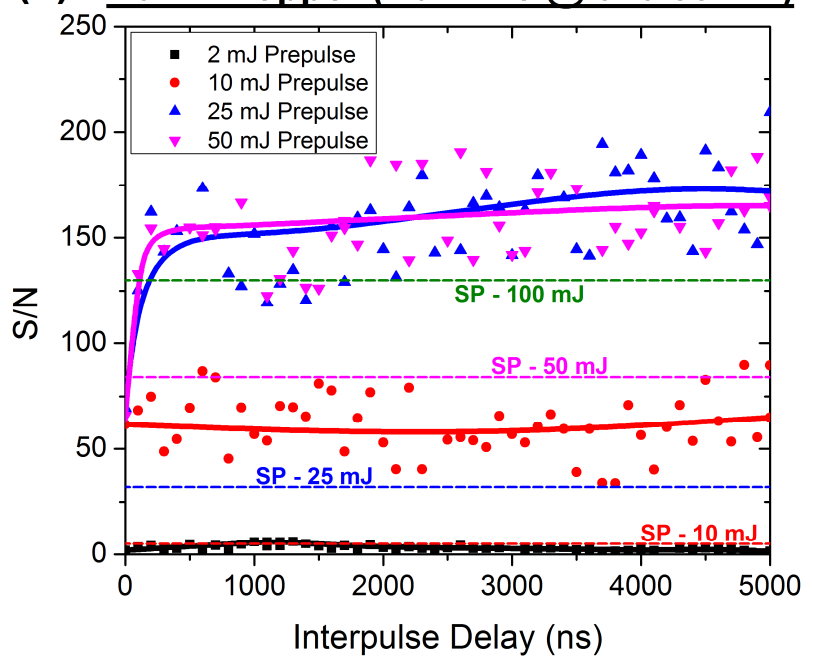

(b) Trace - Iron (Fe I line @ 423.59 nm)

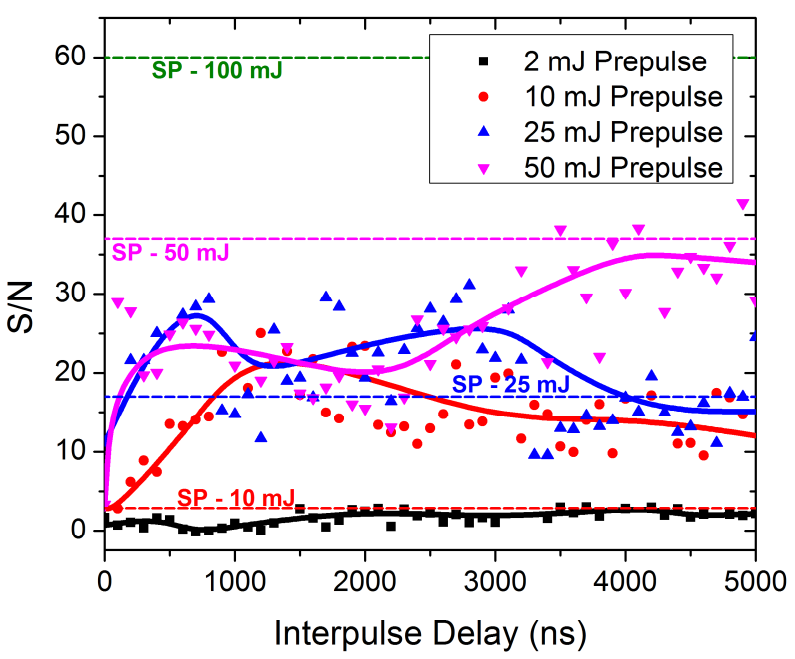

Figure 5 - DP-LIBS: S/N values with respect to interpulse delay for bulk (a) and trace (b) elements under varying prepulse laser energy. Detector gate width was fixed and gate delay was optimized for each prepulse energy according to results in Figure 4. Dashed lines represent optimal SP-LIBS values obtained at respective laser energies. S/B data displayed similar trends and is therefore omitted.

An interesting comparison to highlight is that between SP-LIBS results using a particular laser energy and the DP-LIBS results using that specific laser energy for the first pulse. Since the second heating pulse does not contribute to further sample removal, one is able to quantitatively compare SP- and DP-LIBS results using the same amount of ablated mass and demonstrate the improvement in excitation provided by DP-LIBS. Burdt et al. [30] have shown through experiments, modelling, and comparisons to similar works that proved consistent to theirs, that 
the mass ablation rate is proportional to laser intensity as $\dot{m} \propto I_{L}^{5 / 9}$ for constant laser wavelength and target. As laser intensity is directly proportional to laser energy, the mass ablation rate can be rewritten as $\dot{m} \propto E_{L}^{5 / 9}$ to compare the sample removal rates at different prepulse laser energies. Figure 6 shows the S/N and S/B enhancement factors offered by DP-LIBS compared to SP-LIBS as a function of the relative sample removal rates. Enhancements are shown to be largest for smaller sampled amounts from lower prepulse energies; an important consideration for delicate samples, when sample destruction must be minimized. Without increasing the sampling size and hence sample destruction, one is able to more effectively use the ablated sample and obtain significantly improved results using DP-LIBS and long-wavelength heating. As the quantity of ablated sample increases with increasing prepulse laser energy, the improvement by DP-LIBS becomes less significant. In the case of trace element detection, significant improvements are only observed for the lowest prepulse laser energy.

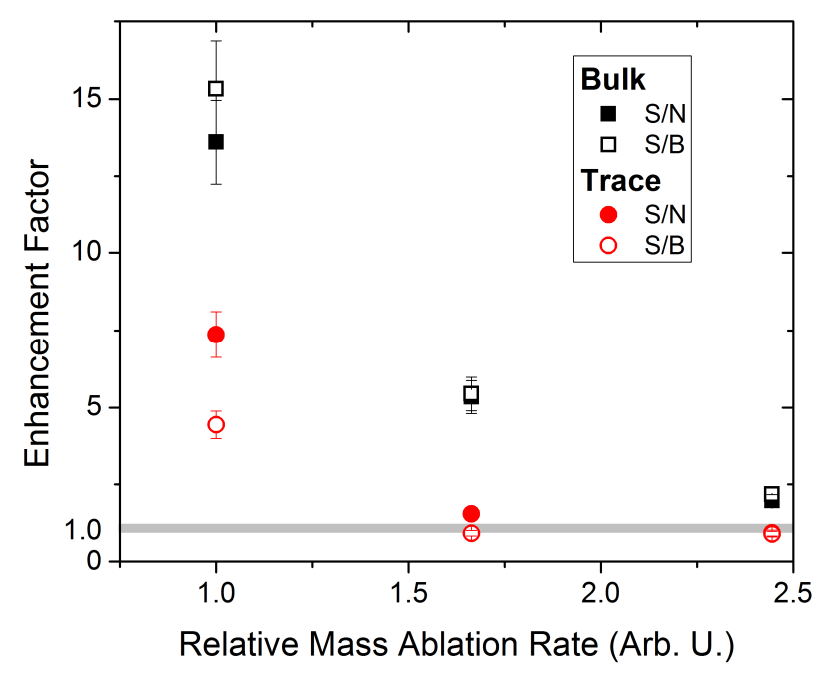

Figure 6 - Enhancement in peak S/N and S/B offered by DP-LIBS compared to SP-LIBS as a function of relative sample ablation rate.

Compared to many other DP-LIBS techniques that employ two Nd:YAG lasers, the cause of improved figures of merit are different, meaning a direct comparison is difficult. 
Enhancements from $1.064 \mu \mathrm{m}-1.064 \mu \mathrm{m}$ (or other Nd:YAG laser harmonics) DP-LIBS are generally due to increased mass ablation, the rarified environment that the second plasma expands into, and heating of the ablated plume, while enhancements from $1.064 \mu \mathrm{m}-10.6 \mu \mathrm{m}$ DP-LIBS are attributed almost entirely to the improved laser-plume coupling and heating of the plume and increased plume persistence. Because of these and differences in laser parameters (wavelength, energy, pulse duration), a quantitative comparison is difficult. Increased background emission has been documented in $1.064 \mu \mathrm{m}-1.064 \mu \mathrm{m}$ DP-LIBS cases [31], but after system optimization the increase in background emission using $1.064 \mu \mathrm{m}-10.6 \mu \mathrm{m}$ DPLIBS was found to be minimal (see reference spectrum in Figure 2). Therefore, from a qualitative standpoint, a clear advantage of the $\mathrm{CO}_{2}$ laser is its ability to offer more efficient use of the ablated mass from the first pulse through improved coupling and absorption without increasing sample destruction while minimizing increases in background emission.

\section{Conclusions}

In this study, the use of long-wavelength heating was explored in DP-LIBS. A traditional SP-LIBS analysis using a $1.064 \mu \mathrm{m}$ Nd:YAG laser was carried out to provide a benchmark for comparison with DP-LIBS results. By using the long-wavelength $\mathrm{CO}_{2}$ laser in DP-LIBS, one is able to efficiently reheat the plume generated by the first laser pulse through inverse bremsstrahlung absorption without increasing the quantity of ablated mass. The use of $\mathrm{S} / \mathrm{N}$ and S/B ratios was found beneficial to incorporate changes in noise and background levels in the analysis and more accurately quantify the signal discrimination ability of the LIBS system. It was found that for bulk elemental analysis, DP-LIBS provided maximum enhancements of about 
14 and 15 times for S/N and S/B, respectively, compared to SP-LIBS using the same quantity of ablated sample. For trace element analysis, maximum enhancements of about 7 and 4 times for S/N and S/B, respectively, were found using DP-LIBS compared to SP-LIBS. Enhancement factors were found to be highest for lower prepulse energies and hence, lower quantities of ablated mass. This is an important consideration for delicate samples, where sample destruction and the quantity of ablated mass must be minimized. As prepulse laser energy increased, the improvements were less substantial, especially for trace elements where only the lowest prepulse energy provided significant improvement.

\section{Acknowledgements}

This work is partially supported by US DOE National Nuclear Security Administration under award number DE-NA0001174 and NSF PIRE.

\section{References}

[1] S.N. Thakur, J.P. Singh, Laser-induced breakdown spectroscopy, 1st ed., Elsevier, Amsterdam ; Boston, 2007.

[2] A.W. Miziolek, V. Palleschi, I. Schechter, Laser-induced breakdown spectroscopy (LIBS) : fundamentals and applications, Cambridge University Press, Cambridge, UK ; New York, 2006.

[3] D.A. Cremers, L.J. Radziemski, Handbook of laser-induced breakdown spectroscopy, John Wiley \& Sons, Chichester, West Sussex, England ; Hoboken, NJ, 2006.

[4] A.P.M. Michel, M. Lawrence-Snyder, S.M. Angel, A.D. Chave, Laser-induced breakdown spectroscopy of bulk aqueous solutions at oceanic pressures: evaluation of key measurement parameters, Appl Optics, 46 (2007) 2507-2515.

[5] B. Salle, D.A. Cremers, S. Maurice, R.C. Wiens, P. Fichet, Evaluation of a compact spectrograph for in-situ and stand-off Laser-Induced Breakdown Spectroscopy analyses of geological samples on Mars missions, Spectrochim Acta B, 60 (2005) 805-815.

[6] D. Anglos, S. Couris, C. Fotakis, Laser diagnostics of painted artworks: Laser-induced breakdown spectroscopy in pigment identification, Appl Spectrosc, 51 (1997) 1025-1030.

[7] J.L. Gottfried, F.C. De Lucia, C.A. Munson, A.W. Miziolek, Laser-induced breakdown spectroscopy for detection of explosives residues: a review of recent advances, challenges, and future prospects, Anal Bioanal Chem, 395 (2009) 283-300.

[8] S. Acquaviva, E. D'Anna, M.L. De Giorgi, F. Moro, Laser-induced breakdown spectroscopy for compositional analysis of multielemental thin films, Spectrochim Acta B, 61 (2006) 810-816. 
[9] L.M. Cabalin, J.J. Laserna, Experimental determination of laser induced breakdown thresholds of metals under nanosecond Q-switched laser operation, Spectrochim Acta B, 53 (1998) 723-730.

[10] J.J. Chang, B.E. Warner, Laser-plasma interaction during visible-laser ablation of methods, Appl Phys Lett, 69 (1996) 473-475.

[11] R.W. Coons, S.S. Harilal, S.M. Hassan, A. Hassanein, The importance of longer wavelength reheating in dual-pulse laser-induced breakdown spectroscopy, Appl Phys B-Lasers O, 107 (2012) 873-880.

[12] J.R. Freeman, S.S. Harilal, P.K. Diwakar, B. Verhoff, A. Hassanein, Comparison of optical emission from nanosecond and femtosecond laser produced plasma in atmosphere and vacuum conditions, Spectrochim Acta B, 87 (2013) 43-50.

[13] D.A. Cremers, A. Beddingfield, R. Smithwick, R.C. Chinni, C.R. Jones, B. Beardsley, L. Karch, Monitoring Uranium, Hydrogen, and Lithium and Their Isotopes Using a Compact LaserInduced Breakdown Spectroscopy (LIBS) Probe and High-Resolution Spectrometer, Appl Spectrosc, 66 (2012) 250-261.

[14] F.R. Doucet, G. Lithgow, R. Kosierb, P. Bouchard, M. Sabsabi, Determination of isotope ratios using Laser-Induced Breakdown Spectroscopy in ambient air at atmospheric pressure for nuclear forensics, J Anal Atom Spectrom, 26 (2011) 536-541.

[15] D.W. Hahn, N. Omenetto, Laser-Induced Breakdown Spectroscopy (LIBS), Part II: Review of Instrumental and Methodological Approaches to Material Analysis and Applications to Different Fields, Appl Spectrosc, 66 (2012) 347-419.

[16] V.I. Babushok, F.C. DeLucia, J.L. Gottfried, C.A. Munson, A.W. Miziolek, Double pulse laser ablation and plasma: Laser induced breakdown spectroscopy signal enhancement, Spectrochim Acta B, 61 (2006) 999-1014.

[17] M. Corsi, G. Cristoforetti, M. Giuffrida, M. Hidalgo, S. Legnaioli, V. Palleschi, A. Salvetti, E. Tognoni, C. Vallebona, Three-dimensional analysis of laser induced plasmas in single and double pulse configuration, Spectrochim Acta B, 59 (2004) 723-735.

[18] X.Y. Pu, N.H. Cheung, ArF laser induced plasma spectroscopy of lead ions in aqueous solutions: Plume reheating with a second Nd : YAG laser pulse, Appl Spectrosc, 57 (2003) 588590.

[19] P.A. Benedetti, G. Cristoforetti, S. Legnaioli, V. Palleschi, L. Pardini, A. Salvetti, E. Tognoni, Effect of laser pulse energies in laser induced breakdown spectroscopy in double-pulse configuration, Spectrochim Acta B, 60 (2005) 1392-1401.

[20] R. Noll, R. Sattmann, V. Sturm, S. Winkelmarm, Space- and time-resolved dynamics of plasmas generated by laser double pulses interacting with metallic samples, J Anal Atom Spectrom, 19 (2004) 419-428.

[21] J. Uebbing, J. Brust, W. Sdorra, F. Leis, K. Niemax, Reheating of a Laser-Produced Plasma by a Second Pulse Laser, Appl Spectrosc, 45 (1991) 1419-1423.

[22] J.R. Freeman, S.S. Harilal, A. Hassanein, B. Rice, Effect of prepulse laser wavelength on EUV emission from CO2 reheated laser-produced Sn plasma, Appl Phys a-Mater, 110 (2013) 853-856.

[23] D.K. Killinger, S.D. Allen, R.D. Waterbury, C. Stefano, E.L. Dottery, Enhancement of Nd : YAG LIBS emission of a remote target using a simultaneous CO2 laser pulse, Opt Express, 15 (2007) 12905-12915. 
[24] M. Weidman, S. Palanco, M. Baudelet, M.C. Richardson, Thermodynamic and spectroscopic properties of Nd:YAG-CO2 Double-Pulse Laser-induced Iron Plasmas, Spectrochim Acta B, 64 (2009) 961-967.

[25] M. Weidman, M. Baudelet, S. Palanco, M. Sigman, P.J. Dagdigian, M. Richardson, Nd:YAG-CO2 double-pulse laser induced breakdown spectroscopy of organic films, Opt Express, 18 (2010) 259-266.

[26] A. Khumaeni, Z.S. Lie, H. Niki, K.I. Fukumoto, T. Maruyama, K. Kagawa, A novel doublepulse laser plasma spectroscopic technique for $\mathrm{H}$ analysis in metal samples utilizing transversely excited atmospheric-pressure CO2 laser-induced metastable He atoms, Opt Rev, 17 (2010) 285289.

[27] J. White, P. Dunne, P. Hayden, F. O'Reilly, G. O'Sullivan, Optimizing $13.5 \mathrm{~nm}$ laserproduced tin plasma emission as a function of laser wavelength, Appl Phys Lett, 90 (2007) 181502.

[28] B.T. Fisher, H.A. Johnsen, S.G. Buckley, D.W. Hahn, Temporal gating for the optimization of laser-induced breakdown spectroscopy detection and analysis of toxic metals, Appl Spectrosc, 55 (2001) 1312-1319.

[29] J.E. Carranza, D.W. Hahn, Sampling statistics and considerations for single-shot analysis using laser-induced breakdown spectroscopy, Spectrochim Acta B, 57 (2002) 779-790.

[30] R.A. Burdt, S. Yuspeh, K.L. Sequoia, Y.Z. Tao, M.S. Tillack, F. Najmabadi, Experimental scaling law for mass ablation rate from a Sn plasma generated by a $1064 \mathrm{~nm}$ laser, J Appl Phys, 106 (2009) 033310.

[31] P.K. Diwakar, S.S. Harilal, J.R. Freeman, A. Hassanein, Role of laser pre-pulse wavelength and inter-pulse delay on signal enhancement in collinear double-pulse laser-induced breakdown spectroscopy, Spectrochim Acta B, 87 (2013) 65-73. 


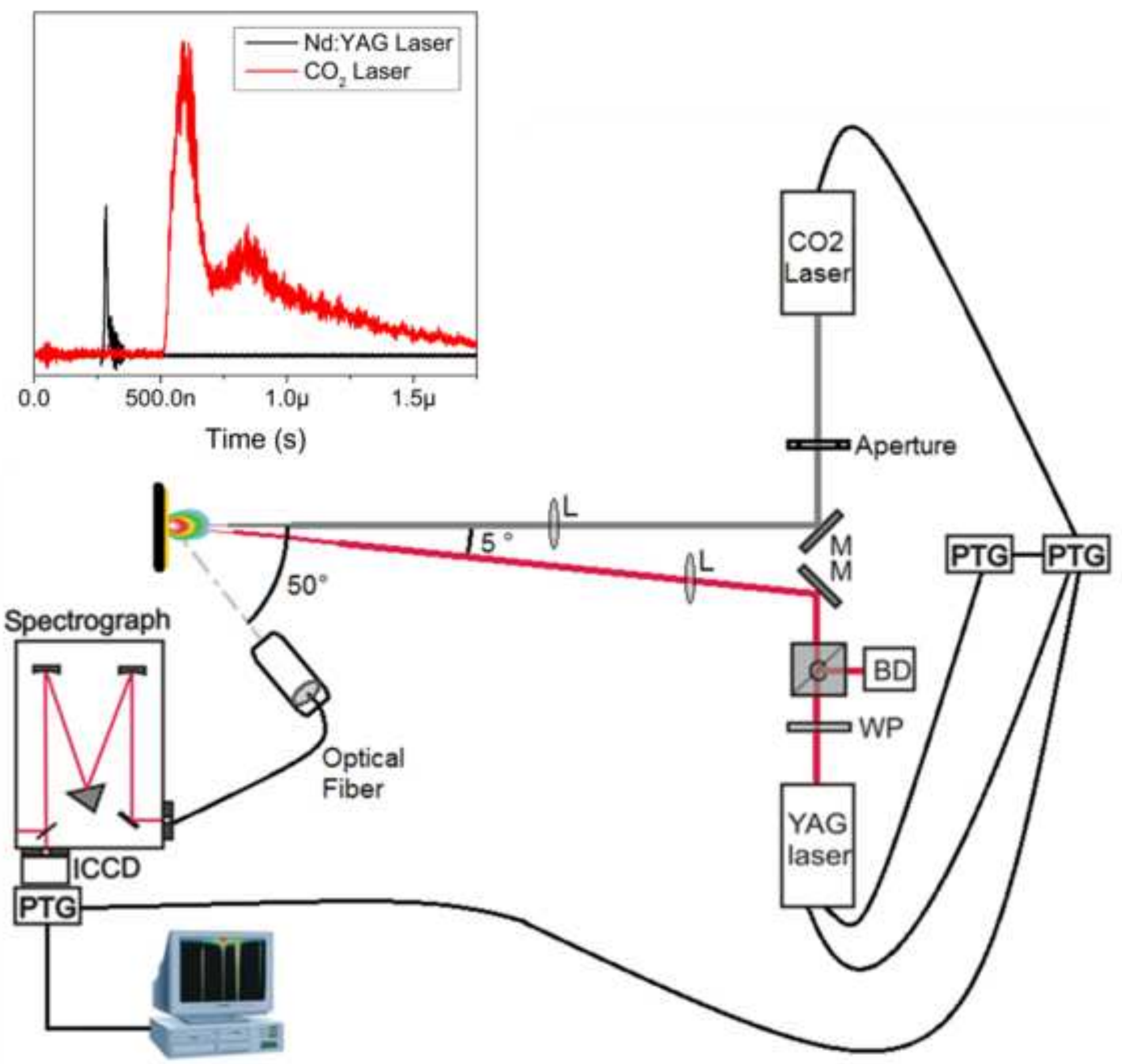




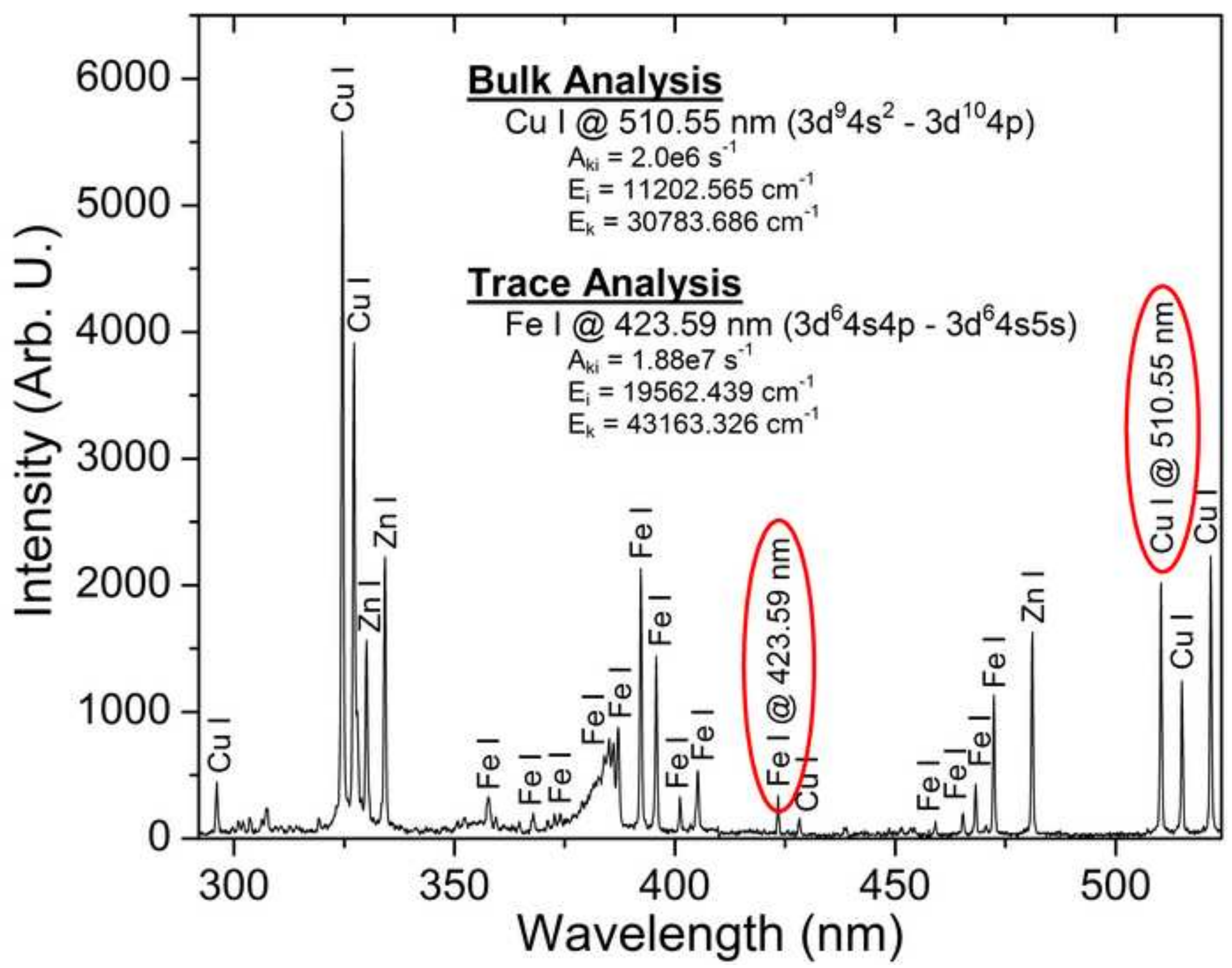




\section{(a) Bulk - Copper (Cu I line @ 510.55 nm}

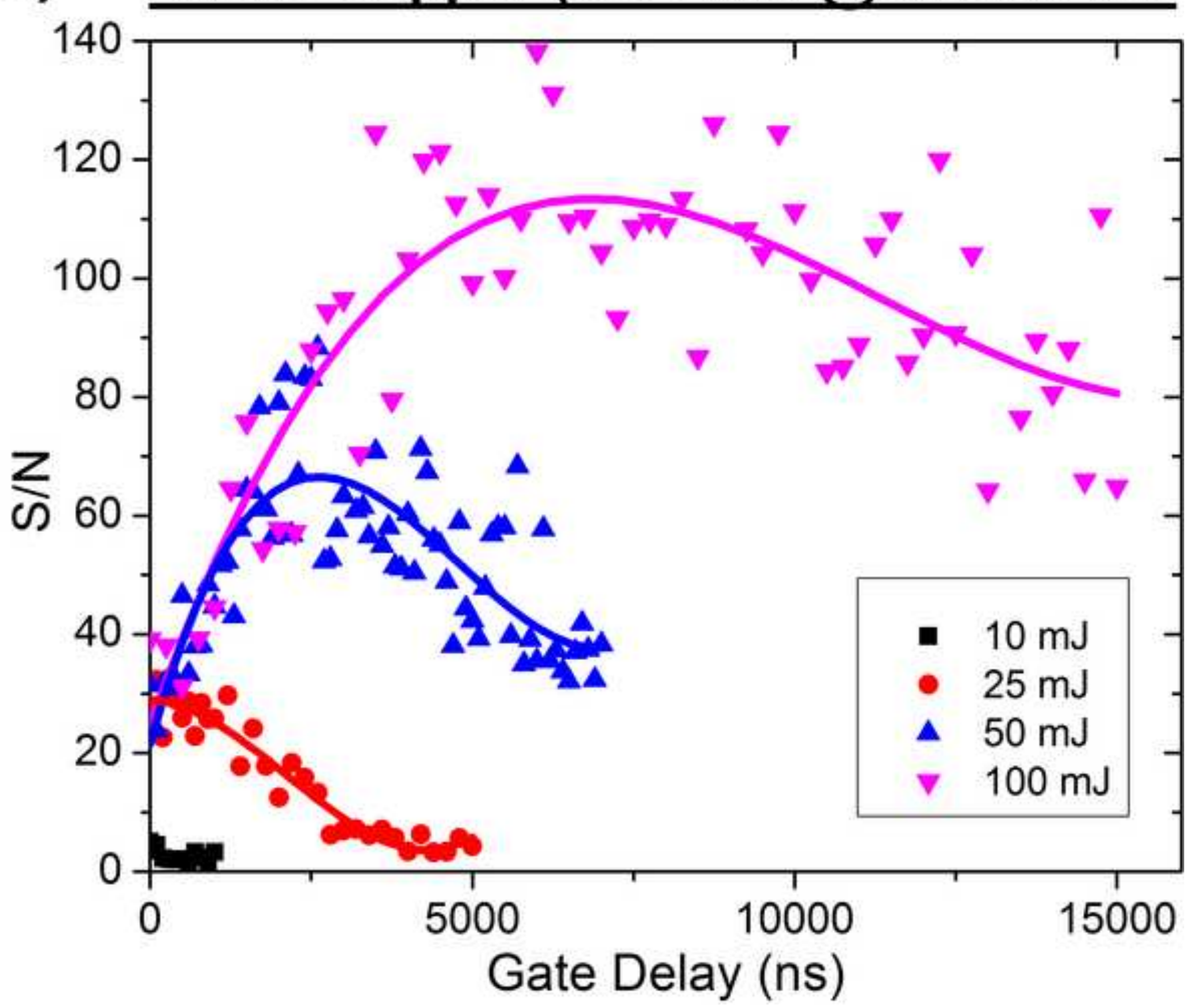


(b) Trace - Iron (Fe I line @ 423.59 nm)

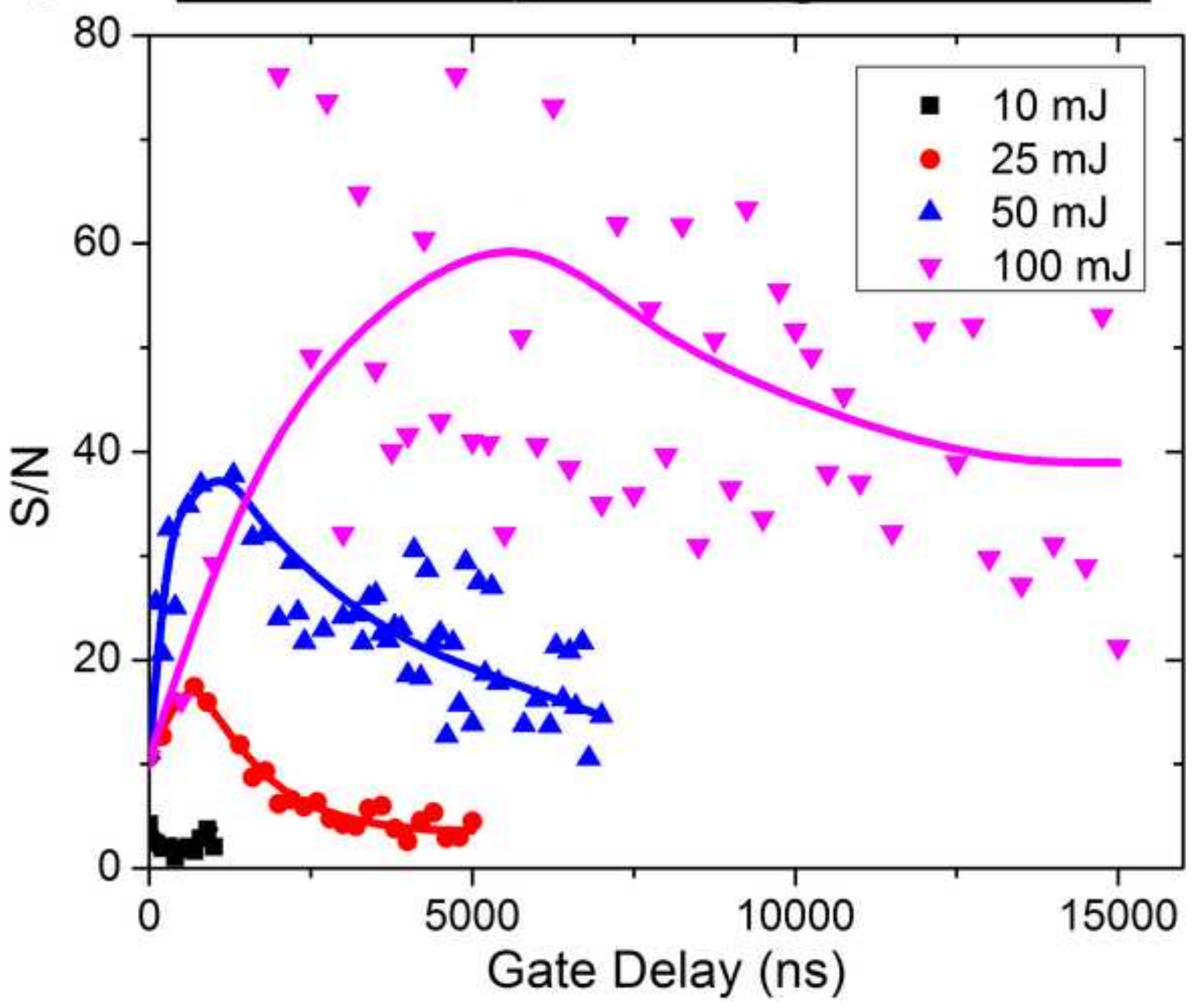




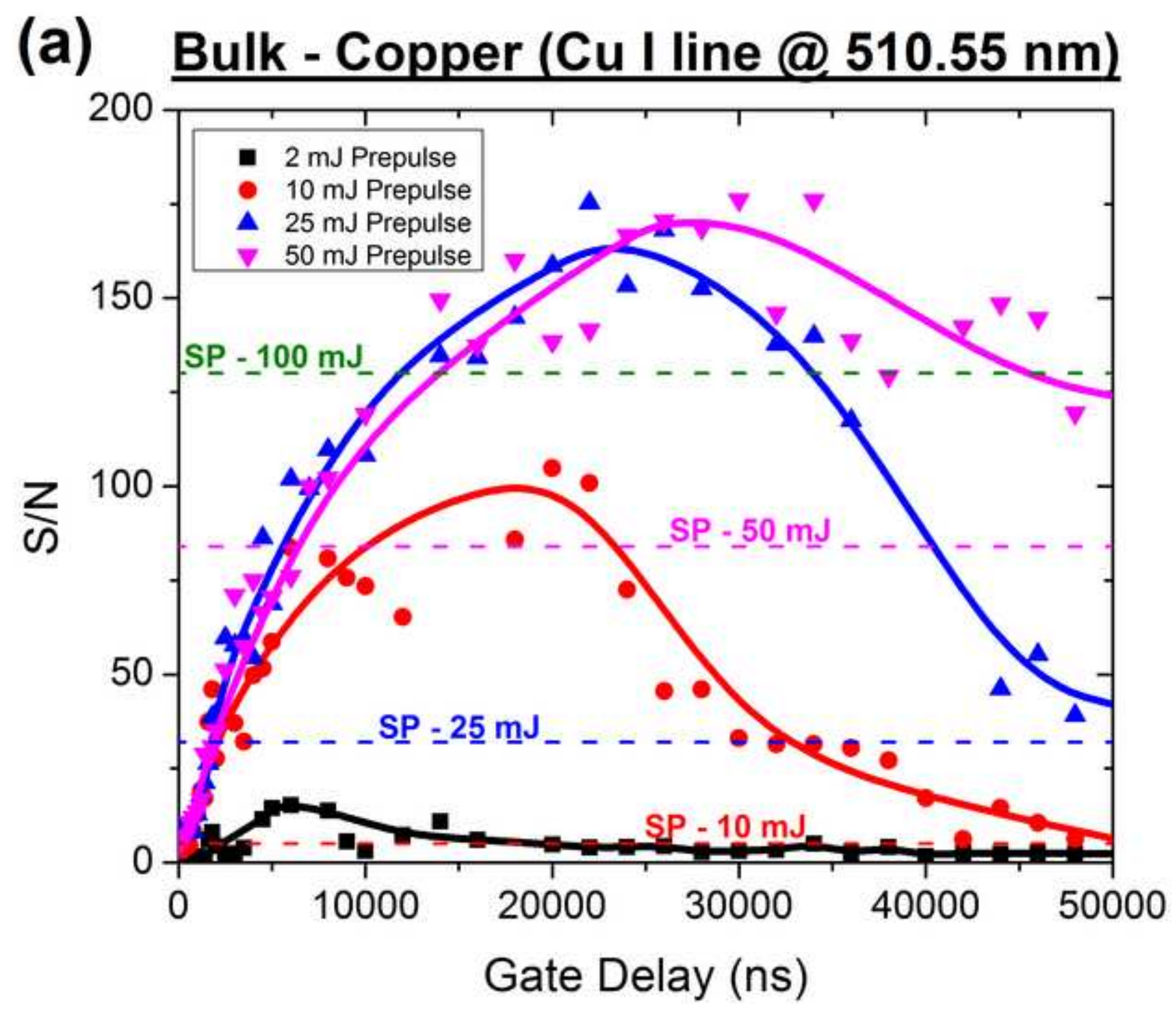




\section{(b) Trace - Iron (Fe I line @ 423.59 nm)}

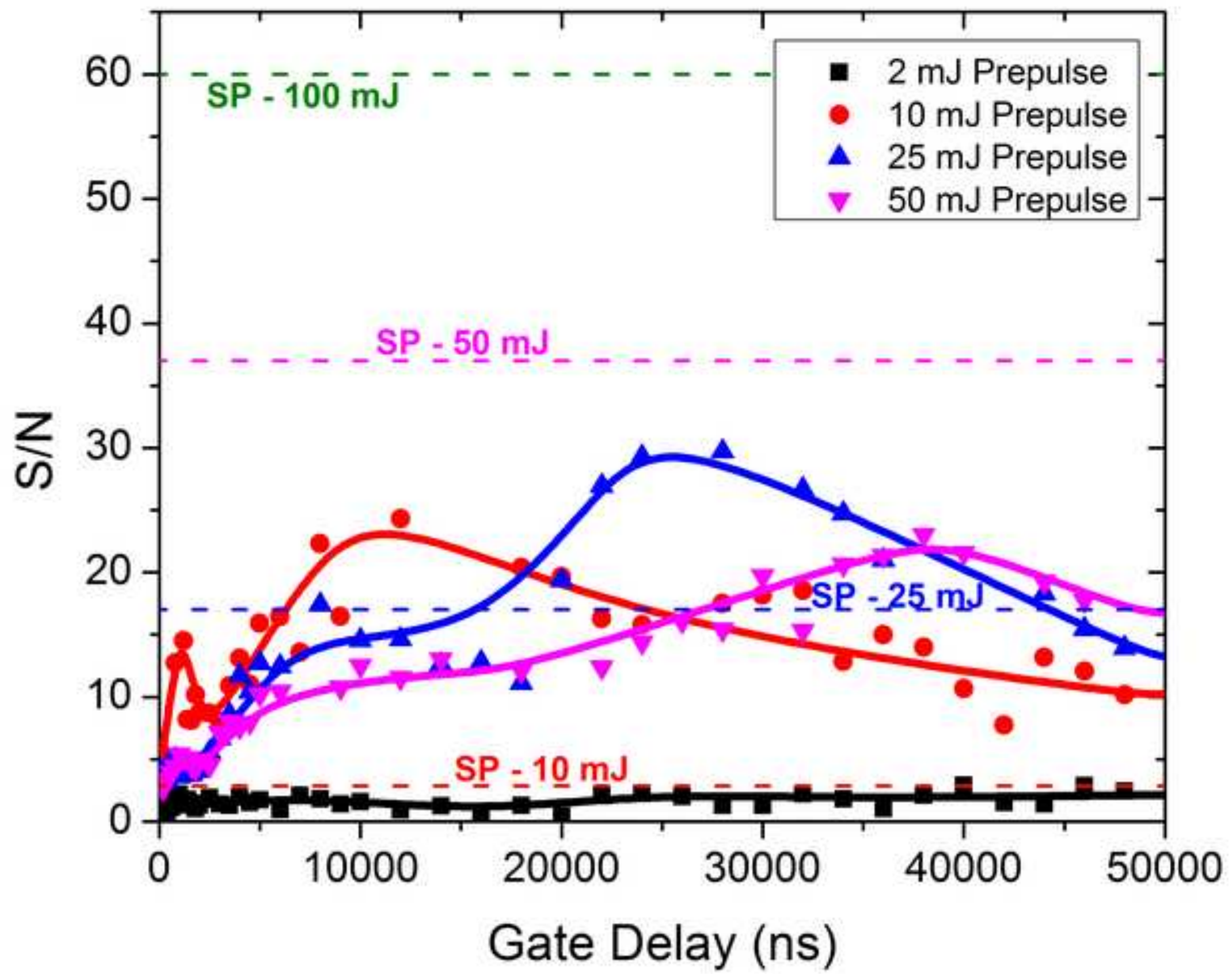




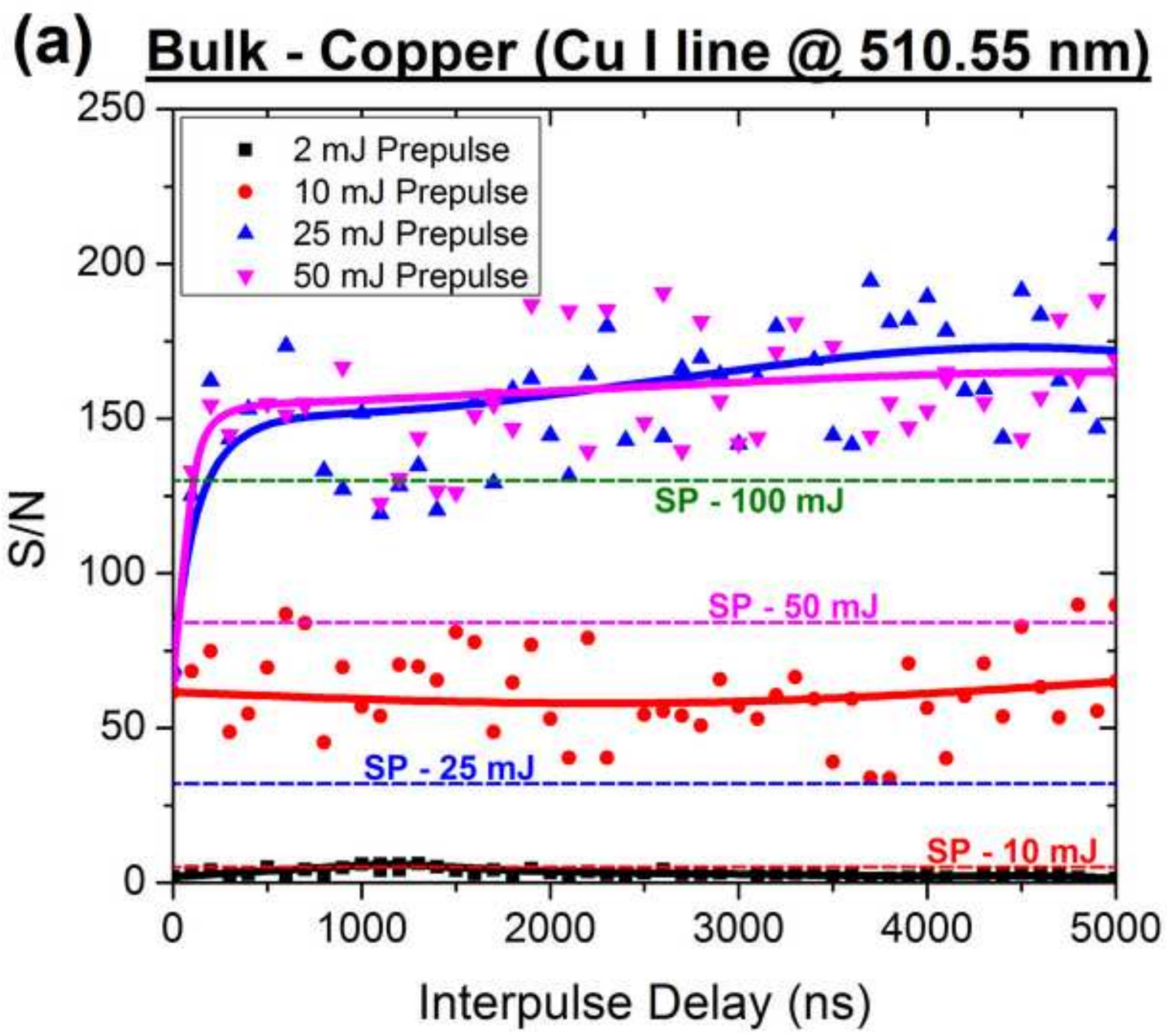


(b) Trace - Iron (Fe I line @ $423.59 \mathrm{~nm}$ )

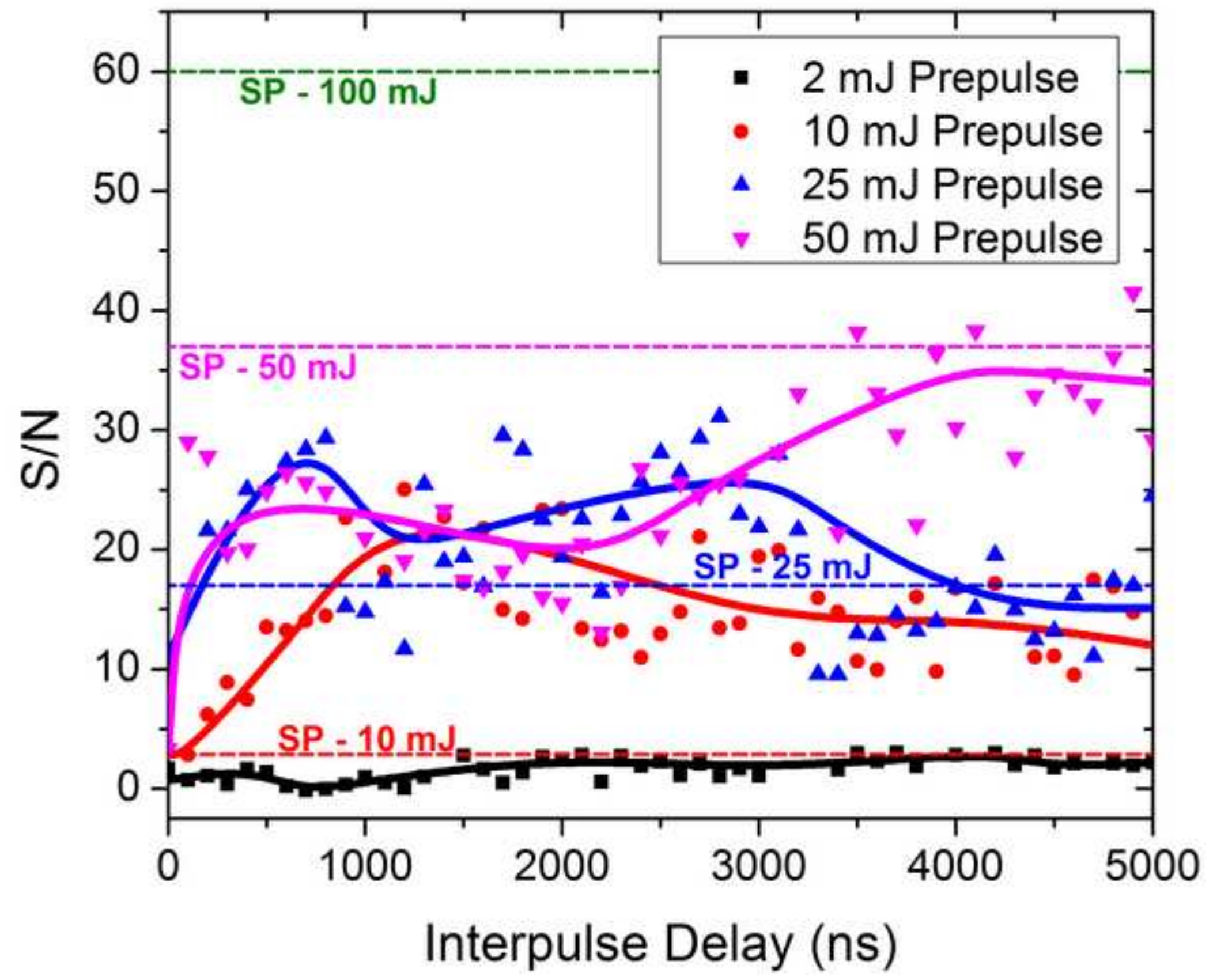




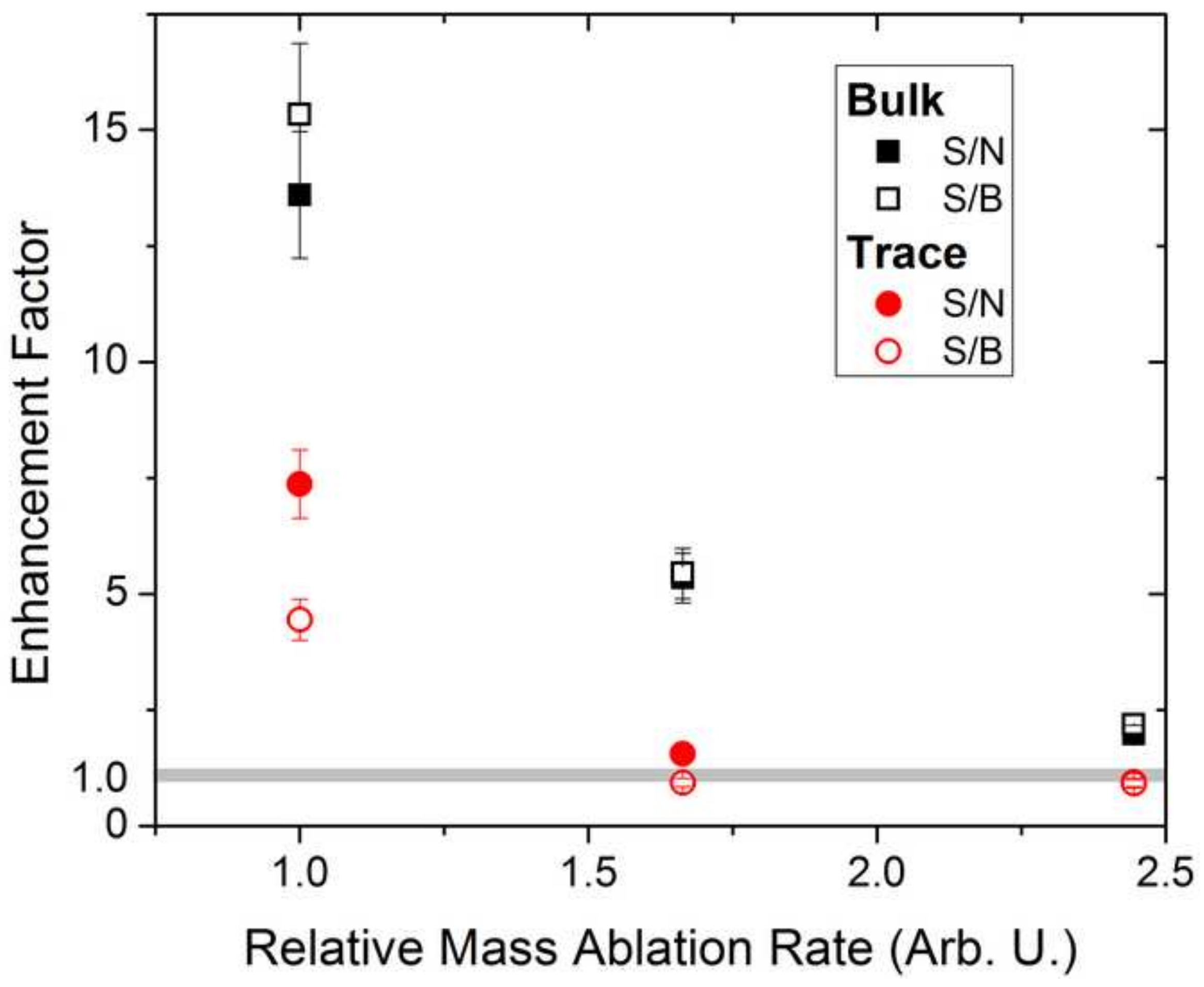

